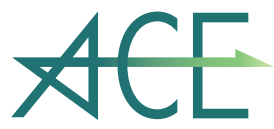

Annals of Clinical Epidemiology

ORIGINAL ARTICLE

\title{
Inhaled nitric oxide for preterm infants with persistent pulmonary hypertension
}

\author{
Takeshi Kimura ${ }^{1}$, Masatoshi Nozaki ${ }^{1}$, Jun Shiraishi ${ }^{1}$, Kazuko Wada $^{1}$, Hiroyuki Kitajima ${ }^{1}$, \\ Neonatal Research Network of Japan
}

\section{ABSTRACT}

\section{BACKGROUND}

Systematic reviews and other studies have not supported the routine use of inhaled nitric oxide (iNO) for preterm infants with respiratory failure. However, despite the lack of evidence, many physicians use iNO for a subgroup of preterm infants with respiratory failure, such as cases of persistent pulmonary hypertension of the newborn (PPHN). This study aimed to clarify whether the use of iNO improved the mortality rate among preterm infants with PPHN.

METHODS

We analyzed data from the Neonatal Research Network in Japan, which is a populationbased nationwide registry. The study included neonates who were born at $<32$ weeks of gestational age between 2006 and 2012 and were diagnosed with PPHN without major congenital malformation including serious congenital heart disease. The primary outcome was the in-hospital mortality rate. Confounder-adjusted odds ratios were estimated using mixed effects logistic regression accounting for clustering within hospitals as random effects and covariates as fixed effects.

RESULTS

We identified 1,231 eligible infants, including 739 infants (60\%) who received iNO. iNO did not significantly improve mortality (odds ratio [OR]: 1.13 , 95\% confidence interval [CI]: $0.82-1.55, P=0.45)$. iNO was significantly associated with bronchopulmonary dysplasia (OR: $1.50,95 \% \mathrm{CI}: 1.11-2.02, P=<0.01$ ) and retinopathy of prematurity requiring treatment (OR: $1.56,95 \%$ CI: $1.11-2.19, P=0.01$ ).

CONCLUSIONS

This retrospective study failed to detect an association between iNO treatment and improved survival among preterm infants with PPHN, however, there are several limitations in this study. Further research is required in this area.

KEY WORDS

low birth weight, mortality, nitric oxide, persistent pulmonary hypertension of the newborn, preterm

\author{
${ }^{1}$ Department of Neonatal Medicine, Osaka \\ Women's and Children's Hospital \\ Corresponding author: Takeshi Kimura \\ Department of Neonatal Medicine, Osaka \\ Women's and Children's Hospital, 840 \\ Murodo-cho, Izumi, Osaka 594-1101, Japan. \\ E-mail: kimura681@gmail.com \\ Received: January 25, 2019 \\ Accepted: March 6, 2019 \\ No.19-05 \\ (C) 2019 Society for Clinical Epidemiology
}




\section{INTRODUCTION}

$\mathrm{P}$ ersistent pulmonary hypertension of the newborn (PPHN) occurs when pulmonary vascular resistance remains abnormally elevated after birth. The reported prevalence of PPHN is 1.9 per 1,000 live births [1]. In these cases, echocardiography reveals right-to-left shunting of blood through the foramen ovale and ductus arteriosus, which leads to severe hypoxemia that may not respond to conventional respiratory support. Among term and late preterm infants with PPHN, inhaled nitric oxide (iNO) improves oxygenation (increment of arterial to alveolar oxygen ratio: $0.10 \pm 0.14$ vs. $0.05 \pm 0.13$ [iNO vs. control]) and reduces the need for extracorporeal membrane oxygenation (relative risk: $0.59)[2,3]$, although, it is not routinely recommended for respiratory failure in preterm infants at $<34$ weeks of gestation $[4,5]$. This is because a small proportion of preterm infants with PPHN developed hypoxic respiratory failure (2.2\%) [6]. A meta-analysis showed an almost significant increase in the incidence of severe intraventricular hemorrhage relative risk: $1.20,95 \%$ CI 0.98-1.47) [7] and some guidelines do not recommend iNO to preterm infants [4]. However, some experts recommend the use of iNO for preterm infants with severe hypoxemia primarily based on the pathophysiology of PPHN [8].

Some observational studies have investigated the efficacy of iNO among preterm infants with PPHN diagnosed using echocardiography, and revealed that iNO improved oxygenation (oxygenation index: $45.1 \pm 22.8$ vs. $18.5 \pm 4.3$ [pre-iNO vs. post-iNO]) and the survival rate (all infants [6/6] treated with iNO survived) $[9,10]$. However, those results are limited by the small number of patients $(n=6-15)$. Furthermore, there are no randomized controlled trials regarding this approach, as the rarity of preterm infants with PPHN makes randomized controlled trials unfeasible. Despite the lack of evidence, many physicians use iNO for preterm infants with severe respiratory failure [11]. The present study aimed to clarify whether iNO improved the mortality rate among preterm infants with PPHN, based on data from a largescale population-based neonatal registry in Japan.

\section{METHODS}

\section{SUBJECT SELECTION}

This study's retrospective protocol was approved by the ethics committee of the Tokyo Women's Medical University. We examined anonymized and de-identified data that were obtained during a population-based cohort study, which was conducted by the Neonatal Research Network in Japan (NRNJ). Informed consent for data collection was obtained from parents when their infants were admitted for treatment. The NRNJ maintains a national database of the clinical characteristics and morbidities of Japanese infants with very low birth weight [12]. The data included $96 \%$ (72/75 as of 2008) of newborns from level 3 neonatal intensive care units (NICU) and from seven level 2 NICUs [13]. Each center also registered all very low birth weight infants and neonates born at $<32$ weeks of gestation who were admitted to the NICU within 28 days after birth.

The present study included neonates who were born at 23 0/7 to $316 / 7$ weeks of gestational age (GA) and diagnosed with PPHN between 2006 and 2012. The diagnosis of PPHN was based on the presence of pulmonary hypertension and right-to-left shunting through the fetal pathway, which was confirmed using echocardiography or clinical findings (i.e., a difference in oxygen saturation between the upper and lower limbs). We excluded newborns with any major congenital malformations, such as serious congenital heart disease or major genetic disorder.

\section{INHALED NITRIC OXIDE}

In Japan, iNO is approved for the treatment of hypoxemic respiratory failure with pulmonary hypertension within 7 days after birth. There was no standard protocol for using iNO to treat PPHN during the study period, although the iNO dose in Japan is usually started at 20 ppm and then gradually tapered based on oxygen saturation [14].

\section{OUTCOMES}

The primary objective of this study was to clarify whether iNO improved the mortality rate among preterm infants ( $<32$ weeks of gestational age) with PPHN. The secondary objective was to evaluate the association between iNO treatment and in-hospital comorbidities, such as bronchopulmonary dysplasia (BPD), patent ductus arteriosus, necrotizing enterocolitis, severe intraventricular hemorrhage, cystic periventricular leukomalacia, or retinopathy of prematurity (ROP) requiring treatment.

\section{DEFINITIONS}

The infants' GA was determined in the following order: (1) an obstetric examination using ultrasonography during the first trimester, (2) an obstetric history that recorded the last menstrual period, or (3) a postnatal physical examination of the infant [15]. Small-for-gestational age was defined as a birth weight that was more than two 
standard deviations below the mean, based on the standard Japanese neonatal anthropometric charts [16]. Antenatal steroid use was defined as the administration of at least one dose of any corticosteroid to accelerate fetal maturity. The presence of BPD at the postmenstrual age of 36 weeks was identified in cases that required oxygen supplementation or positive pressure (positive pressure ventilation or nasal continuous positive airway pressure) [17].

\section{STATISTICAL ANALYSES}

The data were summarized as number and proportion for categorical variables and mean and standard deviations for continuous variables. Categorical variables were compared using Fisher's exact test, and continuous variables were compared using the Wilcoxon rank sum test. Adjusted odds ratio and 95\% confidence intervals were estimated using logistic generalized linear mixed models, which accounted for clustering within hospitals. Restricted maximum likelihood method was used for the covariance and variance estimation. All the following factors were included in the models as potential confounders: GA, birth weight standard deviation score, male sex, antenatal steroid use, histological chorioamnionitis (diagnosed as a Blanc stage of $\geq \mathrm{I}$ ), premature rupture of the membranes, multiple birth, breech presentation at birth, and non-reassuring fetal heart rate. Of these potential confounders, GA and birth weight standard deviation score were treated as continuous variables. Others were handled as categorical variables. We performed multiple imputation of missing covariates by fully conditional specification [18].

Additional post hoc analyses were performed, accounting for immortal time bias, differences in the observation period for each patient, and difference in baseline characteristics. The first set of analyses conducted was a subgroup analysis in infants who survived for more than 7 days. The second set of analyses conducted were multivariable Cox proportional hazards regression analyses to compare mortality between infants with and without iNO. In the Cox model, we adjusted for the same potential confounders as the main analysis. The third set of analyses conducted were propensity score matching and mortality comparison [19]. Infants with and without iNO were matched using a 0.1 standard deviation caliper of the log odds of the propensity score, without replacement.

Two-sided $P$-values of $<0.05$ were considered statistically significant. All analyses were performed using SAS software (version 9.4; SAS Institute, Cary, NC).

\section{RESULTS}

\section{CASE SELECTION}

During the study period, 25,691 neonates were born at 23 $0 / 7$ to $316 / 7$ weeks of GA and registered in the NRNJ database. Among these cases, 1,351 neonates (5\%) were diagnosed with PPHN, although 120 neonates were excluded because of major malformations. Among the remaining 1,231 neonates, iNO was administered to 739 neonates (60\%) and 492 neonates (40\%) were managed without iNO (Fig. 1).

\section{PATIENT CHARACTERISTICS AND OUTCOMES}

The neonates' demographic and perinatal characteristics are shown in Table 1. When we compared the iNO group to the non-iNO group, we observed moderate differences in GA (25.9 weeks vs. 26.7 weeks), birth weight (788 g vs. $865 \mathrm{~g}$ ), antenatal steroid use (54\% vs. $42 \%)$, premature rupture of the membranes (59\% vs. $46 \%$ ), and histological chorioamnionitis ( $59 \%$ vs. $46 \%$ ).

However, no significant difference was observed in the odds of death in the iNO and non-iNO groups (29\% vs. $27 \%, P=0.65)$ (Table 2), even after adjusting for potential confounders (OR: 1.13, 95\% CI: 0.82-1.55) (Table 3). The iNO group had significantly higher odds of BPD at 36 weeks (43\% vs. 31\%; OR: 1.50, 95\% CI: 1.11-2.02, $P \leq 0.01)$ and ROP requiring treatment (36\% vs. $23 \%$; OR: 1.56, 95\% CI: 1.11-2.19, $P=0.01)$. After adjusting for potential confounders, no significant differences were observed in the odds of patent ductus arteriosus ligation, necrotizing enterocolitis, severe intraventricular hemorrhage, or cystic periventricular leukomalacia (Table 3). The proportion of missing values for each variable is described in Supplementary Table 1.

In post hoc analyses, the results of the subgroup who survived over 7 days were not substantially different than the main analysis (Supplementary Table 2, 3). In the multivariable Cox proportional hazards regression analyses, the adjusted hazard ratio of using iNO was 0.96 (95\% CI, 0.74-1.24, $P=0.76)$. The baseline characteristics after propensity score matching are shown in Supplementary Table 4. Even after matching, the odds of death was not significantly different between infants with iNO vs. without iNO (23\% [89/387] vs. $27 \%$ [105/387], $P=0.21)$.

\section{DISCUSSION}

The present study revealed that iNO treatment was not associated with improvements in the rates of morbidities and mortality among preterm infants ( $<32$ weeks of GA) 
with PPHN in a nationally representative Japanese database. In contrast, some small-sample observational studies have indicated that iNO treatment for preterm infants with PPHN was associated with an improved survival rate $[8,9]$, although the present study has a much larger sample size (1,231 cases vs. $6-15$ cases). One was conducted in Canada in 1995 and another was conducted in Australia in 2011. Both studies were case series studies and had no control groups, thus, the evidence level was low.

However, inter-country differences in the survival rate and clinical management of preterm infants may have influenced these conflicting findings. For example, Japan has a lower mortality rate during NICU admissions compared to other countries [20]. Similar to our findings, many previous studies have indicated that iNO treatment does not improve survival among preterm infants without PPHN [5]. Thus, although some physicians use iNO to treat a subset of preterm infants (e.g., those with PPHN) [11], it appears that iNO may only improve their oxygenation, rather than survival.
The iNO group in the present study had significantly increased rates of BPD at 36 weeks and ROP requiring treatment, although previous studies, which included randomized controlled studies investigating the efficacy of iNO to preterm infants (under 37 weeks of GA) with respiratory failure regardless of whether infants had PPHN or not, have not revealed similar findings [5]. The negative effect on BPD in our study may be because of differences in respiratory condition severity between our two groups, as physicians tend to use iNO for infants who have severe pulmonary hypertension or hypoxemia. In previous studies, the iNO group and control group may have had similar respiratory conditions under randomization.

We assume that the negative effect of iNO on ROP was also caused by the high severity of respiratory condition in the iNO group. Long duration of ventilation therapy is reported as a risk factor for ROP [21]. In our study, severe respiratory condition in the iNO group may cause long ventilation duration, and then increase ROP. Theo-

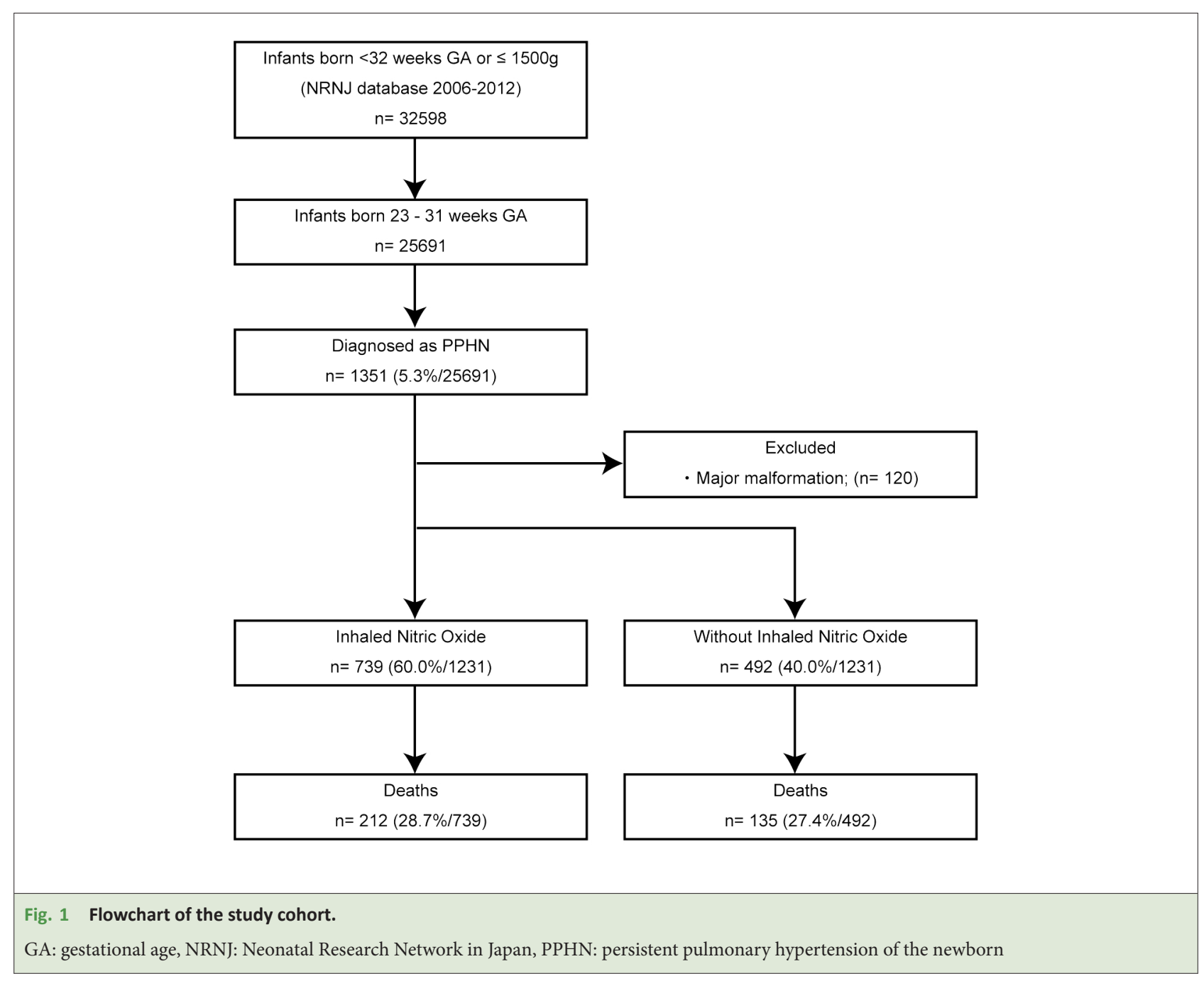


retically, there is a low possibility that iNO directly increases ROP. A previous metanalysis showed no significant relationship between iNO and increased risk of ROP [5].
There are two important limitations in our study. The first limitation is that the database did not include detailed information regarding the iNO treatment, such as the indication, dose, or timing of treatment. In Japan,

\begin{tabular}{|c|c|c|}
\hline Variables & $\begin{array}{l}\text { Infants with iNO } \\
\qquad \mathrm{N}=739\end{array}$ & $\begin{array}{c}\text { Infants without iNO } \\
\qquad \mathrm{N}=492\end{array}$ \\
\hline Gestational age in weeks, mean (SD) & $25.9(2.1)$ & $26.7(2.3)$ \\
\hline 23-27 weeks, n/N (\%) & $602 / 739(81)$ & $344 / 492(70)$ \\
\hline 28-31 weeks, n/N (\%) & $137 / 739(19)$ & $148 / 492(30)$ \\
\hline Birth weight in grams, mean (SD) & $788(259)$ & $865(291)$ \\
\hline$<500 \mathrm{~g}, \mathrm{n} / \mathrm{N}(\%)$ & $71 / 739(10)$ & $38 / 492(8)$ \\
\hline $500-1,000 \mathrm{~g}, \mathrm{n} / \mathrm{N}(\%)$ & $526 / 739(71)$ & $304 / 492(62)$ \\
\hline$>1,000 \mathrm{~g}, \mathrm{n} / \mathrm{N}(\%)$ & $142 / 739(19)$ & $150 / 492(30)$ \\
\hline Small for gestational age, $\mathrm{n} / \mathrm{N}(\%)$ & $106 / 739(14)$ & $89 / 492(18)$ \\
\hline Male sex, n/N (\%) & $386 / 739(48)$ & $274 / 492(56)$ \\
\hline Multiple birth, n/N (\%) & $108 / 739(15)$ & $88 / 492(18)$ \\
\hline Cesarean delivery, $\mathrm{n} / \mathrm{N}(\%)$ & $527 / 739(71)$ & $367 / 492(75)$ \\
\hline Breech presentation, $\mathrm{n} / \mathrm{N}(\%)$ & $259 / 726(36)$ & $170 / 485(35)$ \\
\hline Antenatal steroids, n/N (\%) & $396 / 727(54)$ & $205 / 488(42)$ \\
\hline PROM, n/N (\%) & $434 / 735(59)$ & $219 / 490(45)$ \\
\hline Chorioamnionitis, n/N (\%) & $366 / 623(59)$ & $186 / 404(46)$ \\
\hline Non-reassuring FHR, n/N (\%) & $194 / 704(28)$ & $146 / 483(30)$ \\
\hline Admission at level 3 NICU, n/N (\%) & $656 / 739(89)$ & $435 / 492(88)$ \\
\hline
\end{tabular}

\begin{tabular}{|c|c|c|c|}
\hline Outcomes & Infants with iNO & Infants without iNO & $P$-value \\
\hline Death, n/N (\%) & $212 / 739(29)$ & $135 / 492(27)$ & 0.65 \\
\hline bronchopulmonary dysplasia at 36 weeks, n/N (\%) & $292 / 674(43)$ & $138 / 452(31)$ & $<0.01$ \\
\hline Patent ductus arteriosus ligation, n/N (\%) & $81 / 574(14)$ & $48 / 417(12)$ & 0.25 \\
\hline Necrotizing enterocolitis, n/N (\%) & $30 / 738(4)$ & $18 / 490(4)$ & 0.76 \\
\hline Severe intraventricular hemorrhage, $\mathrm{n} / \mathrm{N}(\%)$ & $160 / 734(22)$ & $96 / 488(20)$ & 0.38 \\
\hline Cystic periventricular leukomalacia, n/N (\%) & $50 / 733(7)$ & $31 / 484(6)$ & 0.81 \\
\hline Retinopathy of prematurity requiring treatment, $\mathrm{n} / \mathrm{N}(\%)$ & $239 / 666(36)$ & $104 / 452(23)$ & $<0.01$ \\
\hline \multicolumn{4}{|c|}{$\begin{array}{l}\text { iNO: inhaled nitric oxide } \\
\text { The denominators were all births. The difference of them is derived from missing values. Proportion of missing value each variable is descri- } \\
\text { bed in Supplementary Table } 1 .\end{array}$} \\
\hline
\end{tabular}


iNO is approved for PPHN only during the first 7 days after birth or after cardiac surgery, although we excluded infants with cardiac malformation requiring surgery. However, some physicians use iNO off-label for preterm infants with chronic respiratory failure caused by BPD or other conditions, and it is possible that some of the patients in the iNO group received treatment for nonPPHN conditions. The second limitation is that unknown confounders may have influenced the results, such as our inability to account for PPHN severity, which was not recorded in the study database. Thus, the iNO group may have had more severe PPHN than the noniNO group. We assumed that the variables we could not obtain due to limitations of the database were respiratory severity (oxygenation index, mean airway pressure, fraction of inspired oxygen) before iNO use, use of vasoactive agent, and primary PPHN etiology. There are some methods to address unmeasured confounders, including randomized controlled trials and instrumental variable methods. The feasibility of randomized controlled trials for the present topic is limited by the small number of preterm infants with PPHN. Furthermore, the clinical course of PPHN is usually rapid, and it would likely be difficult to obtain informed consent from the guardians in that setting. Regarding instrumental variable methods, we could not detect an appropriate variable within the available data in the NRNJ database. Besides unmeasured confounders, there are certain sources of biases in this present study. There could be selection bias. The NRN-J database contains only infants admitted to the NICU, thereby, some infants with PPHN may have died in the delivery room before admission to the NICU. There could also be potential misclassification. There were no definitive guideline regarding treatment of ROP. The indication of ROP was decided by the physician in each institution.

In conclusion, the present study failed to detect an association between iNO treatment and improvement of mortality or survival among preterm infants with PPHN in a nationally representative Japanese registry. However, the present study has several potential sources of bias, such as the absence of detailed information regarding the iNO treatment, and well-designed randomized controlled trials or prospective observational studies are needed to verify our findings.

\section{ACKNOWLEDGMENTS}

The following institutions were enrolled in the NRNJ: Sapporo City General Hospital, Asahikawa Kosei General Hospital, Engaru-Kosei General Hospital, Kushiro Red Cross Hospital, Obihiro-Kosei General Hospital, Tenshi Hospital, NTT Higashinihon Sapporo Hospital, Nikko Memorial Hospital, Nayoro City General Hospital, Sapporo Medical University, Asahikawa Medical University, Aomori Prefectural Central Hospital, Iwate Medical University, Iwate Prefectural Ofunato Hospital, Iwate Prefectural Kuji Hospital, Iwate Prefectural Ninohe Hospital, Sendai Red Cross Hospital, Akita Red Cross Hospital, Tsuruoka Municipal Shonai Hospital, Yamagata University, Yamagata Prefectural Central Hospital, Fukushima Medical University, Takeda General Hospital, Fukushima National Hospital, Tsukuba University, Tsuchiura Kyodo Hospital, Ibaraki Children's Hospital, Dokkyo Medical University, Jichi Medical University, Ashikaga Red Cross Hospital, Gunma Children's Medical Center, Kiryu Kosei General Hospital, Fuji

\begin{tabular}{|c|c|c|c|}
\hline Outcomes & Adjusted OR & $95 \% \mathrm{CI}$ & $P$-value \\
\hline Death & 1.13 & $(0.82-1.55)$ & 0.45 \\
\hline bronchopulmonary dysplasia at 36 weeks & 1.50 & $(1.11-2.02)$ & $<0.01$ \\
\hline Patent ductus arteriosus ligation & 1.06 & $(0.68-1.65)$ & 0.81 \\
\hline Necrotizing enterocolitis & 0.97 & $(0.52-1.80)$ & 0.91 \\
\hline Severe intraventricular hemorrhage & 1.20 & $(0.87-1.65)$ & 0.27 \\
\hline Cystic periventricular leukomalacia & 1.18 & $(0.72-1.95)$ & 0.50 \\
\hline Retinopathy of prematurity requiring treatment & 1.56 & $(1.11-2.19)$ & 0.01 \\
\hline \multicolumn{4}{|c|}{$\begin{array}{l}\text { OR: odds ratio, CI: confidence interval } \\
\text { The adjusted odds ratios were derived from the mixed effects logistic regression models. Hospitals were defined as a random effect. Gesta- } \\
\text { tional age, birth weight standard deviation score, sex, antenatal glucocorticoid exposure, premature rupture of the membranes, multiple } \\
\text { birth, breech presentation at birth, non-reassuring fetal heart rate, and chorioamnionitis were defined as fixed effects. Of these potential } \\
\text { confounders, GA and birth weight standard deviation score were handled as continuous variable. Others were handled as categorical variable. }\end{array}$} \\
\hline
\end{tabular}


Heavy Industries Health Insurance Society Ota Memorial Hospital, Gunma University, Saitama Children's Medical Center, Nishisaitama-chuo National Hospital, Saitama Medical University Saitama Medical Center, Kawaguchi Municipal Medical Center, Jichi Medical University Saitama Medical Center, Asahi General Hospital, Chiba Kaihin Municipal Hospital, Kameda Medical Center, Tokyo Women's Medical University Yachiyo Medical Center, Juntendo University Urayasu Hospital, Tokyo Metropolitan Children's Medical Center, Tokyo Women's Medical University, Aiiku Hospital, Nihon University Itabashi Hospital, National Center for Global Health and Medicine, Tokyo Medical University, Teikyo University, Showa University, Japan Red Cross Medical Center, National Center for Child Health and Development, Tokyo Metropolitan Otsuka Hospital, Toho University, Tokyo Metropolitan Bokuto Hospital, Tokyo Jikei Medical University, Tokyo Medical and Dental University, Saint Luke's International Hospital, Juntendo University, Sanikukai Hospital, Katsushika Red Cross Hospital, Yokohama Rosai Hospital, Yokohama City University Medical Center, St. Marianna University School of Medicine Hospital, Kanagawa Children's Medical Center, Tokai University, Kitazato University, Odawara Municipal Hospital, Nippon Medical School Musashi Kosugi Hospital, Saiseikai Yokohamashi Tobu Hospital, National Hospital Organization Yokohama Medical Center, Yamanashi Prefectural Central Hospital, Nagano Children's Hospital, Shinshu University, Iida Municipal Hospital, National Hospital Organization Shinshu Ueda Medical Center, Saku General Hospital, Niigata University, Niigata Prefectural Central Hospital, Niigata Municipal Hospital, Nagaoka Red Cross Hospital, Koseiren Takaoka Hospital, Toyama Prefectural Central Hospital, Toyama University, Ishikawa Medical Center for Maternal and Child Health, Kanazawa Medical University, Kanazawa Medical Center, Fukui Prefectural Hospital, Fukui University, Gifu Prefectural General Medical Center, National Hospital Organization Nagara Medical Center, Takayama Red Cross Hospital, Seirei Hamamatsu Hospital, Shizuoka Saiseikai Hospital, Shizuoka Children's Hospital, Hamamatsu Medical University, Numazu Municipal Hospital, Yaizu City Hospital, Fujieda Municipal General Hospital, Nagoya Red Cross Daini Hospital, Nagoya University, Nagoya Red Cross Daiichi Hospital, Toyohashi Municipal Hospital, Nagoya City West Medical Center, Anjo Kosei Hospital, Tosei General Hospital, Komaki Municipal Hospital, Toyota Memorial Hospital, Okazaki Municipal Hospital, Konan Kosei Hospital, National Mie Central Medical Center, Ise Red Cross Hospital, Yokkaichi Municipal Hospital, Otsu Red Cross Hospital, Shiga University of Medical Science Hospital, Nagahama Red Cross Hospital, Uji Tokushukai Hospital, The Japan Baptist Hospital, Kyoto Univer- sity, Kyoto Red Cross Daiichi Hospital, National Maizuru Medical Center, Fukuchiyama City Hospital, Kyoto Prefectural University of Medicine Hospital, Kyoto City Hospital, Mitsubishi Kyoto Hospital, Yodogawa Christian Hospital, Osaka Medical Center and Research Institute for Maternal and Child Health, Osaka University, Takatsuki General Hospital, Kansai Medical University, Osaka City General Hospital, Osaka City Sumiyoshi Hospital, Aizenbashi Hospital, Toyonaka Municipal Hospital, National Cerebral and Cardiovascular Center, Kitano Hospital, Saiseikai Suita Hospital, Chifune Hospital, Bell Land General Hospital, Rinku General Medical Center, Osaka Red Cross Hospital, Yao Municipal Hospital, Osaka General Medical Center, Osaka City University, Hyogo Prefectural Kobe Children's Hospital, Kobe University, Kakogawa West City Hospital, Saiseikai Hyogoken Hospital, Kobe City Medical Center General Hospital, Hyogo College of Medicine Hospital, Himeji Red Cross Hospital, Toyooka Public Hospital, Hyogo Prefectural Awaji Medical Center, Nara Medical University, Wakayama Medical University, Tottori Prefectural Central Hospital, Tottori University, Shimane Prefectural Central Hospital, Matsue Red Cross Hospital, Kurashiki Central Hospital, Tsuyama Central Hospital, Kawasaki Medical School Hospital, National Hospital Organization Okayama Medical Center, Okayama Red Cross Hospital, Hiroshima City Hiroshima Citizens Hospital, Hiroshima Prefectural Hospital, Hiroshima University, Tsuchiya General Hospital, National Hospital Organization Kure Medical Center, Yamaguchi University, Yamaguchi Grand Medical Center, Tokushima University, Tokushima Municipal Hospital, Kagawa University, National Hospital Organization Kagawa Children's Hospital, Matsuyama Red Cross Hospital, Ehime Prefectural Central Hospital, Kochi Health Science Center, St. Mary's Hospital, National Kyushu Medical Center, Kurume University, Kitakyushu Municipal Medical Center, University of Occupational and Environmental Health, Fukuoka University, Kyushu University, Iizuka Hospital, National Hospital Organization Kokura Medical Center, National Hospital Organization Saga Hospital, National Hospital Organization Nagasaki Medical Center, Kumamoto City Hospital, Kumamoto University, Oita Prefectural Hospital, Almeida Memorial Hospital, Nakatsu Municipal Hospital, Miyazaki University, National Hospital Organization Miyakonojo Medical Center, Kagoshima City Hospital, Imakiire General Hospital, Okinawa Prefectural Nanbu Medical Center \& Children's Medical Center, Okinawa Prefectural Chubu Hospital, Naha City Hospital, and Okinawa Red Cross Hospital.

\section{DISCLOSURE STATEMENT}

The authors declare no conflict of interest. 
1. Walsh-Sukys MC, Tyson JE, Wright LL, Bauer CR, Korones SB, Stevenson DK, et al. Persistent pulmonary hypertension of the newborn in the era before nitric oxide: practice variation and outcomes. Pediatrics 2000;105:14-20.

2. Neonatal Inhaled Nitric Oxide Study Group. Inhaled nitric oxide in full-term and nearly full-term infants with hypoxic respiratory failure. N Engl J Med 1997;336:597-604. 3. Clark RH, Kueser TJ, Walker MW, Southgate WM, Huckaby JL, Perez JA, et al. Lowdose nitric oxide therapy for persistent pulmonary hypertension of the newborn. Clinical Inhaled Nitric Oxide Research Group. N Engl J Med 2000;342:469-74.

4. Cole FS, Alleyne C, Barks JD, Boyle RJ, Carroll JL, Dokken D, et al. NIH Consensus Development Conference statement: inhaled nitric-oxide therapy for premature infants. Pediatrics 2011;127:363-9.

5. Askie LM, Ballard RA, Cutter GR, Dani C, Elbourne D, Field D, et al. Inhaled nitric oxide in preterm infants: an individualpatient data meta-analysis of randomized trials. Pediatrics 2011;128:729-39.

6. Aikio O, Metsola J, Vuolteenaho R, Perhomaa M, Hallman M. Transient defect in nitric oxide generation after rupture of fetal membranes and responsiveness to inhaled nitric oxide in very preterm infants with hypoxic respiratory failure. J Pediatr 2012;161:397403.e1.

7. Barrington KJ, Finer N, Pennaforte T.
Inhaled nitric oxide for respiratory failure in preterm infants. Cochrane Database of Systematic Reviews 2017;1:CD000509.

8. Kinsella JP, Steinhorn RH, Krishnan US, Feinstein JA, Adatia I, Austin ED, et al. Recommendations for the use of inhaled nitric oxide therapy in premature newborns with severe pulmonary hypertension. J Pediatr 2016;170:312-4.

9. Peliowski A, Finer NN, Etches PC, Tierney AJ, Ryan CA. Inhaled nitric oxide for premature infants after prolonged rupture of the membranes. J Pediatr 1995;126:450-3.

10. Shah DM, Kluckow M. Early functional echocardiogram and inhaled nitric oxide: Usefulness in managing neonates born following extreme preterm premature rupture of membranes (PPROM). J Paediatr Child Health 2011;47:340-5.

11. Ellsworth MA, Harris MN, Carey WA, Spitzer AR, Clark RH. Off-label use of inhaled nitric oxide after release of NIH consensus statement. Pediatrics 2015;135:643-8.

12. Kusuda S, Fujimura M, Sakuma I, Aotani H, Kabe K, Itani Y, et al. Morbidity and mortality of infants with very low birth weight in Japan: center variation. Pediatrics 2006;118:e1130-8.

13. Tokumasu H, Tokumasu S, Kawakami K. Impact of pre-eclampsia in extremely premature infants; A population-based study. Pediatr Int 2016;58:578-83.

14. Omura Y, Otuka N, Saito K, Tanimoto A, Sato M, Yamaguchi T, et al. Inhaled nitric oxide in premature infants with hypoxemic respiratory failure. Journal of Japan Society of Perinatal and Neonatal Medicine 2013;49:1001-6. (in Japanese)

15. Ballard JL, Khoury JC, Wedig K, Wang L, Eilers-Walsman BL, Lipp R. New Ballard Score, expanded to include extremely premature infants. J Pediatr 1991;119:417-23.

16. Itabashi K, Miura F, Uehara R, Nakamura Y. New Japanese neonatal anthropometric charts for gestational age at birth. Pediatr Int 2014;56:702-8.

17. Jobe AH, Bancalari E. Bronchopulmonary dysplasia. Am J Respir Crit Care Med 2001;163:1723-9.

18. van Buuren S. Multiple imputation of discrete and continuous data by fully conditional specification. Stat Methods Med Res 2007;16:219-42.

19. Austin PC, Grootendorst P, Anderson GM. A comparison of the ability of different propensity score models to balance measured variables between treated and untreated subjects: a Monte Carlo study. Stat Med 2007;26:734-53.

20. Shah PS, Lui K, Sjörs G, Mirea L, Reichman B, Adams M, et al. Neonatal outcomes of very low birth weight and very preterm neonates: An international comparison. J Pediatr 2016;177:144-52.e6.

21. Seiberth V1, Linderkamp O. Risk factors in retinopathy of prematurity. a multivariate statistical analysis. Ophthalmologica 2000;214:131-5. 


\begin{tabular}{|lc|}
\hline \multicolumn{1}{|c|}{ Supplementary Table 1 The numbers of missing values } & \\
\hline \multicolumn{1}{|c|}{ Variables } & The proportions of missing data $(\%(\mathrm{n} / \mathrm{N}))$ \\
\hline Gestational age & $0 \%(0 / 1,231)$ \\
Birth weight & $0 \%(0 / 1,231)$ \\
Birth weight standard deviation score & $0 \%(0 / 1,231)$ \\
Sex & $0 \%(0 / 1,231)$ \\
Multiple birth & $0 \%(0 / 1,231)$ \\
Mode of delivery & $0 \%(0 / 1,231)$ \\
Breech presentation & $2 \%(20 / 1,231)$ \\
Antenatal steroids & $1 \%(16 / 1,231)$ \\
Premature rupture of the membranes & $<1 \%(6 / 1,231)$ \\
Chorioamnionitis & $17 \%(204 / 1,231)$ \\
Non-reassuring fetal heart rate & $4 \%(44 / 1,231)$ \\
Admission at level 3 NICU & $0 \%(0 / 1,231)$ \\
Death & $0 \%(0 / 1,231)$ \\
Chronic lung disease at 36 weeks & $9 \%(105 / 1,231)$ \\
Patent ductus arteriosus ligation & $20 \%(240 / 1,231)$ \\
Necrotizing enterocolitis & $<1 \%(3 / 1,231)$ \\
Severe intraventricular hemorrhage & $<1 \%(9 / 1,231)$ \\
Cystic periventricular leukomalacia & $1 \%(14 / 1,231)$ \\
Retinopathy of prematurity requiring treatment & $9 \%(113 / 1,231)$ \\
\hline
\end{tabular}

\begin{tabular}{|c|c|c|c|}
\hline Outcomes & Infants with iNO & Infants without iNO & $P$-value \\
\hline Death, n/N (\%) & $124 / 650(19)$ & $67 / 423(16)$ & 0.19 \\
\hline Bronchopulmonary dysplasia at 36 weeks, $\mathrm{n} / \mathrm{N}$ (\%) & 292/618 (47) & $138 / 397(35)$ & $<0.01$ \\
\hline Patent ductus arteriosus ligation, $\mathrm{n} / \mathrm{N}(\%)$ & $81 / 511(16)$ & $47 / 355(13)$ & 0.33 \\
\hline Necrotizing enterocolitis, n/N (\%) & 28/649 (4) & $15 / 422(4)$ & 0.63 \\
\hline Severe intraventricular hemorrhage, $\mathrm{n} / \mathrm{N}$ (\%) & 129/647 (20) & $71 / 419(17)$ & 0.22 \\
\hline Cystic periventricular leukomalacia, n/N (\%) & $50 / 646(8)$ & $30 / 418(7)$ & 0.81 \\
\hline Retinopathy of prematurity requiring treatment, $\mathrm{n} / \mathrm{N}$ (\%) & 238/607 (39) & $103 / 398(26)$ & $<0.01$ \\
\hline
\end{tabular}




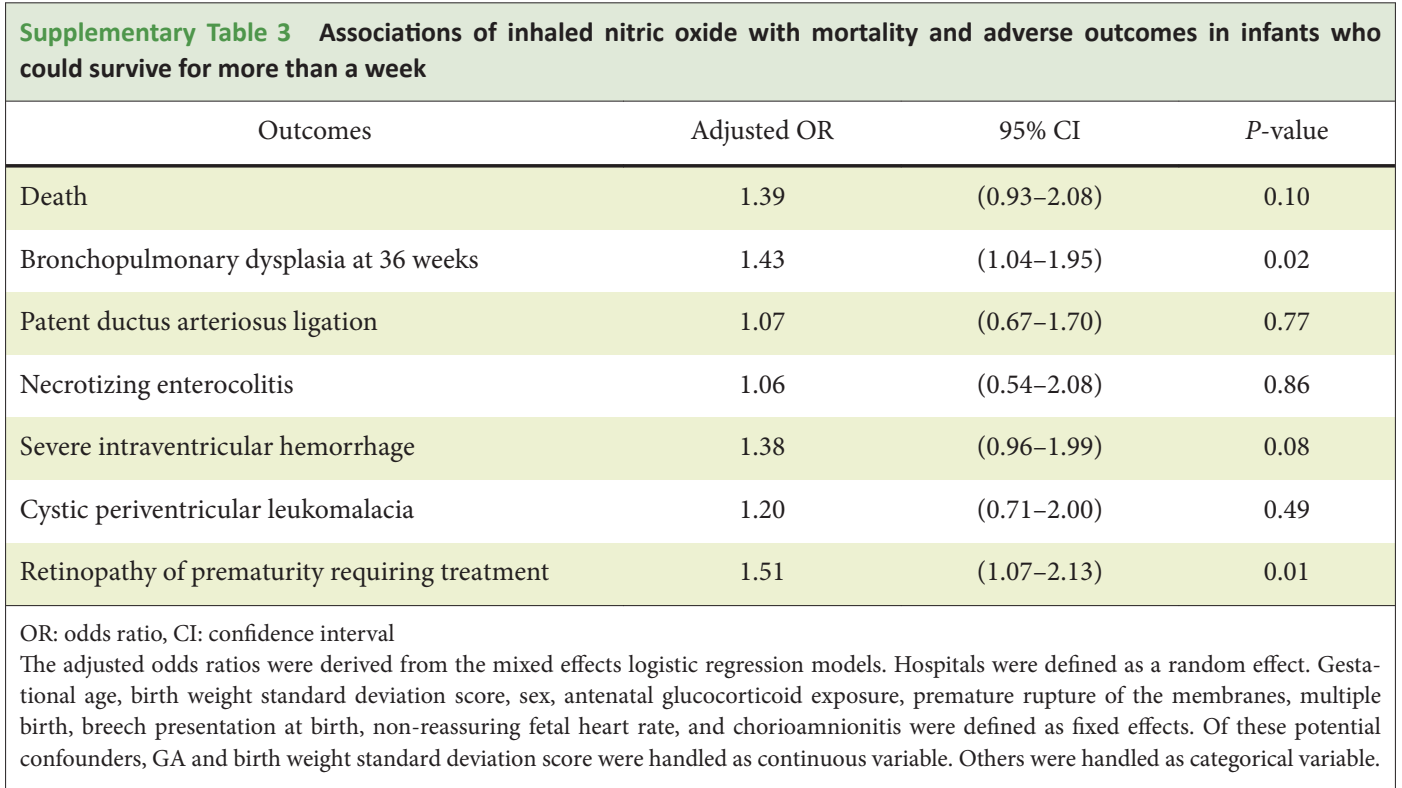

\begin{tabular}{|c|c|c|}
\hline Variables & $\begin{array}{l}\text { Infants with iNO } \\
\qquad \mathrm{N}=387\end{array}$ & $\begin{array}{c}\text { Infants without iNO } \\
\qquad \mathrm{N}=387\end{array}$ \\
\hline Gestational age in weeks, mean (SD) & $25.9(2.2)$ & $26.6(2.3)$ \\
\hline Birth weight standard deviation score, mean (SD) & $-0.5(1.3)$ & $-0.6(1,3)$ \\
\hline Male sex, $\mathrm{n} / \mathrm{N}(\%)$ & $218 / 387(56)$ & $206 / 387(53)$ \\
\hline Multiple birth, n/N (\%) & $50 / 387(13)$ & $69 / 387(18)$ \\
\hline Antenatal steroids, $\mathrm{n} / \mathrm{N}(\%)$ & $207 / 387(53)$ & $174 / 387(45)$ \\
\hline Premature rupture of the membranes, $\mathrm{n} / \mathrm{N}(\%)$ & $227 / 387(59)$ & $175 / 387(45)$ \\
\hline Chorioamnionitis, $\mathrm{n} / \mathrm{N}(\%)$ & $230 / 387(59)$ & $180 / 387(47)$ \\
\hline Non-reassuring fetal heart rate, $\mathrm{n} / \mathrm{N}(\%)$ & $98 / 387(25)$ & $113 / 387(29)$ \\
\hline
\end{tabular}

